\title{
Comparison of pulse pressure variation and pleth variability index in the prone position in pediatric patients under 2 years old
}

\author{
Sang-Hwan Ji ${ }^{1}$, In-Kyung Song ${ }^{2}$, Young-Eun Jang ${ }^{1}$, Eun-Hee Kim ${ }^{1}$, \\ Ji-Hyun Lee ${ }^{1}$, Jin-Tae Kim ${ }^{1}$, and Hee-Soo Kim ${ }^{1}$ \\ Department of Anesthesiology and Pain Medicine, ${ }^{1}$ Seoul National University Hospital, Seoul National University \\ College of Medicine, ${ }^{2}$ Asan Medical Center, University of Ulsan College of Medicine, Seoul, Korea
}

\begin{abstract}
Background: The assessment of intravascular volume status is very important especially in children during anesthesia. Pulse pressure variation (PPV) and pleth variability index (PVI) are well known parameters for assessing intravascular volume status and fluid responsiveness. We compared PPV and PVI for children aged less than two years who underwent surgery in the prone position.

Methods: A total of 27 children were enrolled. We measured PPV and PVI at the same limb during surgery before and after changing the patients' position from supine to prone. We then compared PPV and PVI at each period using Bland-Altman plot for bias between the two parameters and for any correlation. We also examined the difference between before and after the position change for each parameter, along with peak inspiratory pressure, heart rate and mean blood pressure.

Results: The bias between PPV and PVI was $-2.2 \%$ with a $95 \%$ limits of agreement of $-18.8 \%$ to $14.5 \%$, not showing significant correlation at any period. Both PPV and PVI showed no significant difference before and after the position change.

Conclusions: No significant correlation between PVI and PPV was observed in children undergoing surgery in the prone position. Further studies relating PVI, PPV, and fluid responsiveness via adequate cardiac output estimation in children aged less than 2 years are required.
\end{abstract}

Keywords: Arterial pressure; Child; Fluid therapies; Plethysmography; Prone positions.

Corresponding author: Hee-Soo Kim, M.D., Ph.D.

Department of Anesthesiology and Pain Medicine, Seoul National University Hospital, Seoul National University College of Medicine, 101 Daehak-ro, Jongno-gu, Seoul 03080, Korea

Tel: +82-2-2072-3659, Fax: +82-2-747-8364

Email: dami0605@snu.ac.kr

ORCID: https://orcid.org/0000-0002-2661-7944

Received: April 3, 2019.

Revised: May 30, 2019.

Accepted: June 16, 2019.

Korean J Anesthesiol 2019 October 72(5): 466-471 https://doi.org/10.4097/kja.19128

\section{Introduction}

Respiration-induced arterial pressure variation (RIAPV), which is superior to the traditional parameters for assessing intravascular volume status, refers to the variation in arterial pressure that occurs during positive-pressure mechanical ventilation and is regarded as an indicator of 'fluid responsiveness' [1]. Different parameters have been developed to quantify the RIAPV, represented by pulse pressure variation (PPV) or systolic pressure variation (SPV). Recently, the pleth variability index (PVI) has been developed commercially for non-invasive monitoring of plethysmographic variation. Several groups have investigated

(c) This is an open-access article distributed under the terms of the Creative Commons Attribution Non-Commercial License (http://creativecommons.org/ licenses/by-nc/4.0/), which permits unrestricted non-commercial use, distribution, and reproduction in any medium, provided the original work is properly cited. 
whether PVI is a useful tool for assessing hemodynamics, but the results remain controversial [2-5].

Prior studies evaluating the value of PPV or plethysmographic indices such as PVI to predict fluid responsiveness have been predominantly performed in adult patients undergoing major thoracic or abdominal surgery or under intensive care with a pulmonary artery catheter [6,7]. Some of these studies have indicated that plethysmographic indices are useful for assessing fluid responsiveness $[6,8,9]$. This approach is appealing as it allows continuous measurement of fluid responsiveness without invasive methods such as arterial pressure-derived indices.

Two recent studies examined various parameters for predicting fluid responsiveness in pediatric patients, using the aortic blood flow velocity time integral as a gold standard for identifying volume responders. A study [10] presented only $\Delta \mathrm{V}_{\text {peak }}$ (respiratory variation in aortic blood flow velocity) as a predictor of fluid responsiveness, whereas the other [4] showed that both PVI and $\Delta \mathrm{V}_{\text {peak }}$ were effective in identifying fluid responders and non-responders, with a recommended cut-off value of $11 \%$ for PVI. PPV did not prove to be a predictor of fluid responsiveness, probably because of differences in arterial compliance between adults and children [10,11].

Position change during surgery is believed to be a major factor affecting hemodynamic stability $[12,13]$; hence, more thorough monitoring is mandatory. In addition, many studies have reported that the position of patients can affect both their hemodynamics and the data provided by various monitors [14-16]. However, it is very difficult to make echocardiographic evaluations of patients undergoing surgery in the prone position, therefore making $\Delta \mathrm{V}_{\text {peak }}$ unobtainable. In this case, anesthesiologists can refer to PVI or PPV as an alternative to $\Delta \mathrm{V}_{\text {peak }}$, though not satisfactory. For adult patients, Biais et al. [17] reported that PPV can predict fluid responsiveness in the prone position with a cut-off value of $15 \%$.

In this study, we evaluated the relationship between PVI and PPV in the supine and prone positions in young children aged less than 2 years to evaluate whether there is a correlation between these two parameters.

\section{Materials and Methods}

\section{Study population}

The study was approved by the Institutional Review Board (H-1501-075-641) and registered at http://cris.nih.go.kr (KCT0001613). After obtaining informed consent from the parents or legal guardians, pediatric patients aged less than 2 years who underwent neurosurgery in the prone position under general anesthesia and invasive blood pressure monitoring were enrolled. The exclusion criteria were a history or presence of peripheral vascular disease, disorders of the cardiovascular or central nervous system, or other hemodynamically unstable status of patients.

\section{Study protocol}

On the day of surgery, patients arrived in the operating theater after appropriate fasting and without premedication. Monitoring of lead II of a three-lead electrocardiogram, noninvasive blood pressure at $1 \mathrm{~min}$ intervals, peripheral pulse oximetry $\left(\mathrm{SpO}_{2}\right)$, end-tidal carbon dioxide and body temperature was performed using a patient monitor (Solar 8000, GE Medical, USA). Anesthesia was induced in children with an intravenous (IV) injection of 5-6 mg/kg of sodium thiopental and $0.6 \mathrm{mg} / \mathrm{kg}$ of rocuronium after loss of consciousness. We maintained anesthesia with $100-200 \mu \mathrm{g} / \mathrm{kg} / \mathrm{min}$ of propofol and $0.1-0.5 \mu \mathrm{g} / \mathrm{kg} / \mathrm{min}$ of remifentanil. Arterial cannulation was performed for invasive blood pressure monitoring using a 24-gauge JELCO ${ }^{\circledR}$ IV catheter (Smiths Medical, USA). PVI monitoring was started using a Masimo rainbow SET ${ }^{\circledR}$ (Masimo, USA). PVI and $\mathrm{SpO}_{2}$ were monitored at the same limb with arterial cannulation. After confirming that the vital signs had stabilized, the patient's data were transmitted to a personal computer using an analog-to-digital converter (DA 149, DATAQ Instruments, USA) for 1 min without stimulating the patient. After the patient was moved to the prone position and the patient's vital signs had stabilized, the data were obtained in the same manner. During data transmission, we adjusted the anesthetic depth to maintain the bispectral index between 40 and 60 . No specific changes to the rate of fluid or anesthetics administration were made throughout these periods. The mode of mechanical ventilation was maintained as volume-controlled ventilation with tidal volume of $8-10 \mathrm{ml} / \mathrm{kg}$ and positive end-expiratory pressure (PEEP) of $0-5 \mathrm{cmH}_{2} \mathrm{O}$ according to clinical need. Both tidal volume and PEEP were kept stationary before and after the position change.

\section{Calculation of parameters}

Pulse pressure (PP) is the difference between systolic pressure (SP) and diastolic pressure (DP); that is,

$$
\mathrm{PP}=\mathrm{SP}-\mathrm{DP}
$$

PPV is defined as the difference between the maximum and minimum PP over a single respiratory cycle. It is calculated as follows:

$$
\mathrm{PPV}=\left(\left[\mathrm{PP}_{\max }-\mathrm{PP}_{\min }\right] / \mathrm{PP}_{\text {mean }}\right) \times 100 \%
$$

Perfusion index (PI) is the ratio of the pulsatile signal to the 
non-pulsatile signal obtained from the pulse plethysmograph. It is calculated as follows:

$$
\mathrm{PI}=(\mathrm{AC} / \mathrm{DC}) \times 100
$$

where AC indicates a variable amount of light absorbed by the pulsatile arterial flow and DC is a constant amount of light from the pulse oximeter. To reflect respiratory variations in PI, PVI is calculated as follows:

$$
\mathrm{PVI}=100 \times\left(\mathrm{PI}_{\max }-\mathrm{PI}_{\min }\right) / \mathrm{PI}_{\max }
$$

\section{Data analysis}

PPV data based on arterial blood pressure were obtained for $1 \mathrm{~min}$ at $10 \mathrm{~s}$ intervals for both periods, that are after induction of anesthesia with supine position (period 1) and 1 min after the change in position to prone (period 2). PPV was calculated for each period automatically, using computer software (Vital recorder, VitalDB team, Seoul National University College of Medicine, Korea) [18] that automatically records patients' vital signs and calculates parameters. Calculations were made for six
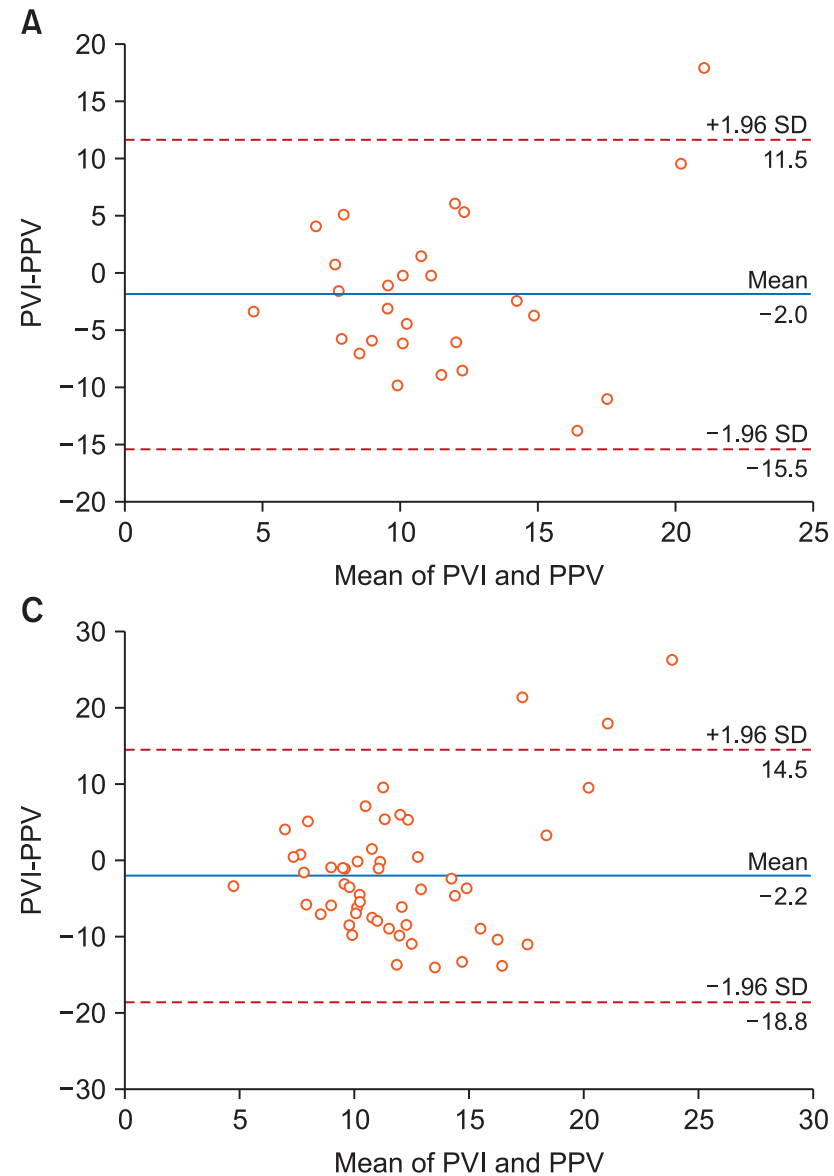

explicit sub-periods of $10 \mathrm{~s}$ without overlap. PPV values were averaged for each period before being regarded as final. PVI data were recorded at the end of each period. Peak inspiratory pressure, heart rate and mean blood pressure were also recorded for each period.

\section{Sample size calculation}

In a pilot study, the mean of difference was $32.6 \%$ with a standard deviation of $30.4 \%$ with an upper limit of 195.8 and a lower limit of -130.7 . With these data, and $\alpha=0.05, \beta=0.2$, the required sample size was 6 pairs. As the required size was comparatively small, we enrolled patients based on a previous

Table 1. Demographic Characteristics

\begin{tabular}{lc}
\hline Age (months) & $7.2 \pm 4.7$ \\
Height $(\mathrm{cm})$ & $70.0 \pm 6.7$ \\
Weight $(\mathrm{kg})$ & $8.6 \pm 1.6$ \\
Anesthesia time (min) & $271.2 \pm 115.4$ \\
Sex $(\mathrm{M}: \mathrm{F})$ & $17: 10$ \\
\hline
\end{tabular}

Values are presented as mean $\pm \mathrm{SD}$.

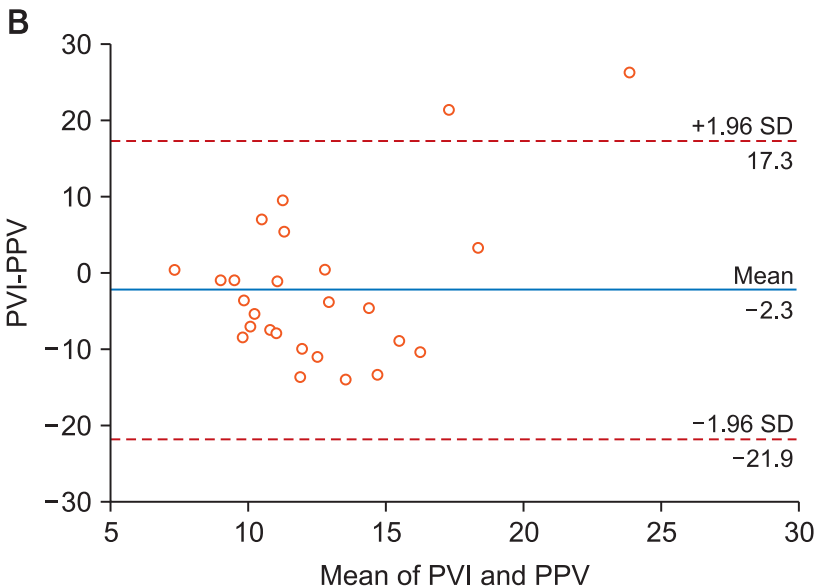

Fig. 1. Bland-Altman plot comparing PPV and PVI. PPV and PVI were compared for supine position (A), prone position (B) and altogether (C). PPV: pulse pressure variation, PVI: pleth variability index. 
similar study, which included 24 [3]. In total, 27 patients were enrolled.

\section{Statistical analysis}

After excluding the effect of artifacts, mean values of PPV were calculated for each period. A fast-flush test [19] was performed to identify and remove cases with overdamped arterial waveform. As some evidence suggests that PVI can predict fluid responsiveness $[4,5]$, we regarded PVI as the gold standard, since there were no better alternative. PPV and PVI were compared using a Bland-Altman plot and the correlation coefficient between PPV and PVI was calculated.

Each parameter before and after the change in position from supine to prone (periods 1 and 2) was compared using a paired $t$-test. Statistical analyses were performed using SPSS version 22.0 (SPSS Inc., USA) and MedCalc ${ }^{\circledR}$ version 17.2 (MedCalc Software, Belgium). P values of $<0.05$ were considered statistically significant.

\section{Results}

A total of 34 patients were screened and 31 patients completed the study; 27 datasets were collected, with four cases discarded because of incomplete data. Demographic data are shown in Table 1.

In the supine position, the correlation coefficient between PPV and PVI was 0.130 with a P value of 0.518 . The bias between PPV and PVI was $-2.0 \%$, with 95\% limits of agreement of $-15.5 \%$ to $11.5 \%$. In the prone position, the correlation coefficient between PPV and PVI was -0.371 with a P value of 0.062 . The bias between PPV and PVI was $-2.3 \%$, with $95 \%$ limits of agreement of $-21.9 \%$ to $17.3 \%$. Altogether, the correlation coefficient between PPV and PVI was -0.131 with a P value of 0.350 . The bias between PPV and PVI was $-2.2 \%$, with 95\% limits of agreement of $-18.8 \%$ to $14.5 \%$ (Fig. 1). According to the paired t-test, both PPV and PVI showed no statistically significant difference before and after the position change from supine to prone ( $\mathrm{P}=0.245,0.535$, respectively). There was no statistically significant difference in PEEP and peak inspiratory pressure before and after the change in position to prone $(\mathrm{P}=0.064,0.162$, respectively). The heart rate showed a statistically significant difference following the position change, while the mean blood pressure did not $(\mathrm{P}=0.000,0.879$, respectively) (Table 2 ).

\section{Discussion}

The correlation between PPV and PVI was insignificant in both the positions and overall; hence, one cannot replace the other. The bias seems acceptable, but the range of $95 \%$ limits of
Table 2. Comparison of Period 1 (Supine) and Period 2 (Prone) for Pulse Pressure Variability, Pleth Variability Index, Peak Inspiratory Pressure, Heart Rate, and Mean Blood Pressure

\begin{tabular}{llrrr}
\hline & & Supine & Prone & P value \\
\hline PPV (\%) & Mean & 12.63 & 13.97 & 0.245 \\
& SD & 4.51 & 5.42 & \\
& SD/Mean & 0.36 & 0.39 & \\
PVI (\%) & Mean & 10.1 & 10.88 & 0.535 \\
& SD & 5.64 & 7.06 & \\
& SD/Mean & 0.56 & 0.65 & \\
PIP $\left(\mathrm{cmH}_{2} \mathrm{O}\right)$ & Mean & 19.81 & 20.52 & 0.162 \\
& SD & 3.52 & 3.07 & \\
HR (beats/min) & Mean & 154.81 & 145.71 & $0.000^{*}$ \\
& SD & 16.81 & 19.02 & \\
MBP (mmHg) & Mean & 64.04 & 63.52 & 0.879 \\
& SD & 9.10 & 18.60 & \\
\hline
\end{tabular}

PPV: pulse pressure variation, PVI: pleth variability index, PIP: peak inspiratory pressure, HR: heart rate, MBP: mean blood pressure, SD: standard deviation. ${ }^{*} \mathrm{P}<0.05$.

agreement in the supine and prone positions is highly unacceptable in each position and overall.

A previous study [3] on children undergoing spinal fusion showed similar findings with the present study and reported that PVI is not a surrogate for PPV, and that PVI measurements were not affected by a change in position from supine to prone, and therefore PVI may be a useful parameter in patients undergoing spine surgery. We obtained similar results in a younger population.

As the difference in PPV following position change is not statistically significant, we can infer that increased intrathoracic pressure in the prone position does not substantially affect PPV in young children. Further, RIAPV does not seem to increase much in this circumstance [20].

This study has several limitations. First, automatic recording of PPV carries a risk of artifacts at any time, especially immediately after a position change. Although we attempted to exclude the effect of these artifacts as mentioned in the methods section, we admit that our efforts could be imperfect and subjective. PVI, however, was recorded manually only once at the end of each period. Like PPV, it would have been better if we recorded PVI multiple times for each period.

Second, light absorption can be affected by many factors such as the site of measurement, the structure of peripheral vasculature and the degree of peripheral vasoconstriction. The site for PVI measurement is diverse in small children. For example, PVI could be measured at the digits in some larger children, whereas it could be measured at the palm in some smaller infants. This can lower the reliability of PVI in small children. A recent study showed that PVI measurements in neonates are poorly reproducible, even within a single limb [21]. 
Third, we could not assess fluid responsiveness in children in this study owing to the position of the patients. To assess fluid responsiveness, we would need an additional means of measurement to directly or indirectly estimate the cardiac output. In a previous study from our institution, transthoracic echocardiography was used to indirectly assess volume status and thus fluid responsiveness [4], which is impossible in patients in the prone position. Otherwise, we should have used arterial pressure waveform analysis [22] or pulmonary artery catheterization for the real-time measurement of cardiac output, which are both unsuitable for children aged less than two years. If it had been possible to measure fluid responsiveness, we would have divided the children into responder and non-responder groups. We might also be able to evaluate the accuracy of PPV and PVI measurements with a gold standard.

Finally, we did not strictly control the ventilatory profile for each patient. Dynamic parameters such as PPV and PVI can be affected by intrathoracic pressure, especially during positive-pressure ventilation. Although our study mainly focused on the comparison of PPV and PVI at the same time, it would have been better if we controlled the tidal volume and PEEP and evaluated the effect of the ventilatory profile by subgroup analysis.

In conclusion, the results of our study suggest that there is little correspondence between PVI and PPV for children less than two years of age undergoing a position change from supine to prone. Neither PPV nor PVI showed a significant change following a position change from supine to prone. Further study comparing PPV and PVI with respect to fluid responsiveness via adequate cardiac output estimation is required to improve our strategy for fluid administration management in young children aged less than two years.

\section{Conflicts of Interest}

No potential conflict of interest relevant to this article was reported.

\section{Author Contributions}

Sang-Hwan Ji (Formal analysis; Validation; Writing - original draft; Writing-review \& editing)

In-Kyung Song (Conceptualization; Data curation; Formal analysis; Writing-original draft)

Young-Eun Jang (Data curation; Methodology; Writing-review \& editing)

Eun-Hee Kim (Data curation; Methodology; Writing-review \& editing)

Ji-Hyun Lee (Data curation; Formal analysis; Writing-review \& editing)

Jin-Tae Kim (Conceptualization; Data curation; Supervision; Writing-review \& editing)

Hee-Soo Kim (Conceptualization; Supervision; Writing-review \& editing)

\section{ORCID}

Sang-Hwan Ji, https://orcid.org/0000-0001-6736-4464

In-Kyung Song, https://orcid.org/0000-0002-7699-2005

Young-Eun Jang, https://orcid.org/0000-0002-7511-4104

Eun-Hee Kim, https://orcid.org/0000-0003-0697-1935

Ji-Hyun Lee, https://orcid.org/0000-0002-8384-8191

Jin-Tae Kim, https://orcid.org/0000-0002-3738-0081

Hee-Soo Kim, https://orcid.org/0000-0002-2661-7944

\section{References}

1. Michard F. Changes in arterial pressure during mechanical ventilation. Anesthesiology 2005; 103: 419-28.

2. Chandler JR, Cooke E, Petersen C, Karlen W, Froese N, Lim J, et al. Pulse oximeter plethysmograph variation and its relationship to the arterial waveform in mechanically ventilated children. J Clin Monit Comput 2012; 26: 145-51.

3. Feldman JM, Sussman E, Singh D, Friedman BJ. Is the pleth variability index a surrogate for pulse pressure variation in a pediatric population undergoing spine fusion? Paediatr Anaesth 2012; 22: 250-5.

4. Byon HJ, Lim CW, Lee JH, Park YH, Kim HS, Kim CS, et al. Prediction of fluid responsiveness in mechanically ventilated children undergoing neurosurgery. Br J Anaesth 2013; 110: 586-91.

5. Renner J, Broch O, Gruenewald M, Scheewe J, Francksen H, Jung O, et al. Non-invasive prediction of fluid responsiveness in infants using pleth variability index. Anaesthesia 2011; 66: 582-9.

6. Grassi P, Lo Nigro L, Battaglia K, Barone M, Testa F, Berlot G. Pulse pressure variation as a predictor of fluid responsiveness in mechanically ventilated patients with spontaneous breathing activity: a pragmatic observational study. HSR Proc Intensive Care Cardiovasc Anesth 2013; 5: 98-109.

7. Oliveira-Costa CD, Friedman G, Vieira SR, Fialkow L. Pulse pressure variation and prediction of fluid responsiveness in patients ventilated with low tidal volumes. Clinics (Sao Paulo) 2012; 67: 773-8.

8. Yang SY, Shim JK, Song Y, Seo SJ, Kwak YL. Validation of pulse pressure variation and corrected flow time as predictors of fluid responsiveness in patients in the prone position. Br J Anaesth 2013; 110: 713-20. 
9. Michard F, Lopes MR, Auler JO Jr. Pulse pressure variation: beyond the fluid management of patients with shock. Crit Care 2007; $11: 131$.

10. Pereira de Souza Neto E, Grousson S, Duflo F, Ducreux C, Joly H, Convert J, et al. Predicting fluid responsiveness in mechanically ventilated children under general anaesthesia using dynamic parameters and transthoracic echocardiography. Br J Anaesth 2011; 106: 856-64.

11. Durand P, Chevret L, Essouri S, Haas V, Devictor D. Respiratory variations in aortic blood flow predict fluid responsiveness in ventilated children. Intensive Care Med 2008; 34: 888-94.

12. Pump B, Talleruphuus U, Christensen NJ, Warberg J, Norsk P. Effects of supine, prone, and lateral positions on cardiovascular and renal variables in humans. Am J Physiol Regul Integr Comp Physiol 2002; 283: R174-80.

13. Dharmavaram S, Jellish WS, Nockels RP, Shea J, Mehmood R, Ghanayem A, et al. Effect of prone positioning systems on hemodynamic and cardiac function during lumbar spine surgery: an echocardiographic study. Spine (Phila Pa 1976) 2006; 31: 1388-93.

14. Brown ZE, Görges M, Cooke E, Malherbe S, Dumont GA, Ansermino JM. Changes in cardiac index and blood pressure on positioning children prone for scoliosis surgery. Anaesthesia 2013; 68: 742-6.

15. Luostarinen T, Lindroos AC, Niiya T, Silvasti-Lundell M, Schramko A, Hernesniemi J, et al. Prone versus sitting position in neurosurgerydifferences in patients' hemodynamic management. World Neurosurg 2017; 97: 261-6.

16. Xia J, Yuan J, Lu X, Yin N. Prone position results in enhanced pressor response to ephedrine compared with supine position during general anesthesia. J Clin Anesth 2016; 31: 94-100.

17. Biais M, Bernard O, Ha JC, Degryse C, Sztark F. Abilities of pulse pressure variations and stroke volume variations to predict fluid responsiveness in prone position during scoliosis surgery. Br J Anaesth 2010; 104: 407-13.

18. Lee HC, Jung CW. Vital Recorder-a free research tool for automatic recording of high-resolution time-synchronised physiological data from multiple anaesthesia devices. Sci Rep 2018; 8: 1527.

19. Kleinman B, Powell S, Kumar P, Gardner RM. The fast flush test measures the dynamic response of the entire blood pressure monitoring system. Anesthesiology 1992; 77: 1215-20.

20. Marks R, Silverman R, Fernandez R, Candiotti KA, Fu E. Does the systolic pressure variation change in the prone position? J Clin Monit Comput 2009; 23: 279-82.

21. den Boogert WJ, van Elteren HA, Goos TG, Reiss IKM, de Jonge RCJ, van den Berg VJ. Reproducibility of the Pleth Variability Index in premature infants. J Clin Monit Comput 2018; 32: 457-64.

22. Marik PE, Cavallazzi R, Vasu T, Hirani A. Dynamic changes in arterial waveform derived variables and fluid responsiveness in mechanically ventilated patients: a systematic review of the literature. Crit Care Med 2009; 37: 2642-7. 